\title{
WITTEN VOLUME FORMULAS FOR SEMI-SIMPLE LIE ALGEBRAS
}

\author{
JIANQIANG ZHAO
}

\begin{abstract}
In this paper we provide an algebraic derivation of the explicit Witten volume formulas for a few semi-simple Lie algebras by combining a combinatorial method with the ideas used by Gunnells and Sczech in computation of higher-dimensional Dedekind sums.
\end{abstract}

\section{INTRODUCTION}

In [8] Witten related the volumes of the moduli spaces of representations of the fundamental groups of two dimensional surfaces to the special values of the following zeta function attached to complex semisimple Lie algebras $\mathfrak{g}$ at positive integers:

$$
\zeta_{W}(s ; \mathfrak{g})=\sum_{\varphi} \frac{1}{(\operatorname{dim} \varphi)^{s}}
$$

where $\varphi$ runs over all finite dimensional irreducible representations of $\mathfrak{g}$. By physics consideration Witten showed that for any positive integer $m$

$$
\zeta_{W}(2 m ; \mathfrak{g})=c(2 m ; \mathfrak{g}) \pi^{2 m r},
$$

where $c(2 m ; \mathfrak{g}) \in \mathbb{Q}$ and $r$ is the number positive roots of $\mathfrak{g}$. Such formulas are now called Witten volume formula.

The precise Witten volume formula for $\mathfrak{s l}(3)$ was obtained by Zagier [9] (and independently by Garoufalidis and Weinstein):

$$
\zeta_{W}(2 m ; \mathfrak{s l}(3))=\frac{4^{m+1}}{3} \sum_{\substack{0 \leq i \leq 2 m \\
i \equiv 0 \bmod 2}}\left(\begin{array}{c}
4 m-i-1 \\
2 m-1
\end{array}\right) \zeta(i) \zeta(6 m-i) .
$$

In [1], Gunnells and Sczech studied higher-dimensional Dedekind sums and established their reciprocity law. As one of the applications they could derived the Witten volume formula for $\mathfrak{s l}(4)$ precisely.

Matsumoto and his collaborators recently defined the multiple variable analogs of $\zeta_{W}(s ; \mathfrak{g})$ and studied some of their analytical and arithmetical properties (see [2, 3, 4, 6]):

$$
\zeta_{\mathfrak{g}}\left(\left\{s_{\alpha}\right\}_{\alpha \in \Delta_{+}}\right):=\sum_{m_{1}, \ldots, m_{\ell}=1}^{\infty} \prod_{\alpha \in \Delta_{+}}\left\langle\alpha^{\vee}, m_{1} \lambda_{1}+\cdots+m_{\ell} \lambda_{\ell}\right\rangle^{-s_{\alpha}}
$$

where for fixed set $\Delta=\left\{\alpha_{1}, \ldots, \alpha_{\ell}\right\}$ of fundamental roots $\Delta_{+}$is set of all positive roots of $\mathfrak{g}$, $\alpha^{\vee}=2 \alpha /\langle\alpha, \alpha\rangle$ is the coroot, and $\left\{\lambda_{1}, \ldots, \lambda_{\ell}\right\}$ are the fundamental weights such that $\left\langle\alpha_{i}^{\vee}, \lambda_{j}\right\rangle=\delta_{i, j}$. 
By simple computation

$$
\zeta_{W}(s ; \mathfrak{g})=M(\mathfrak{g})^{s} \zeta_{\mathfrak{g}}(s, \ldots, s), \quad \text { where } \quad M(\mathfrak{g})=\prod_{\alpha \in \Delta_{+}}\left\langle\alpha^{\vee}, \lambda_{1}+\cdots+\lambda_{\ell}\right\rangle .
$$

With this multiple variable setup Matsumoto et al. were able to obtain more general formulas which include Witten volume formulas as special cases for Lie algebras such as $\mathfrak{s o}(5)$ and $\mathfrak{g}_{2}$. However, their computation involves complicated analytical tools.

In this paper, we combine our combinatorial method developed in [10, 11, 12] and the technique of Gunnells and Sczech to provide an algebraic proof of Witten volume formulas for $\mathfrak{s o}(5)$ and $\mathfrak{g}_{2}$.

This paper is inspired by the work of Gunnells and Sczech [1]. I want to thank them for their detailed explanation of the part of their paper closely related to Witten zeta functions.

\section{THE KEY IDEAS}

We briefly recall the setup in [1, $\S 1$. Let $L$ be a lattice of $\operatorname{rank} \ell \geq 1$ and $L^{*}=\operatorname{Hom}_{\mathbb{Z}}(L, \mathbb{Z})$. Denote by 0 the zero linear form in $L^{*}$. Let $r \geq \ell$. For $e=\left(e_{1}, \ldots, e_{r}\right) \in \mathbb{N}^{r}, v \in L^{*} \otimes \mathbb{R}$ and $\sigma=\left(\sigma_{1}, \ldots, \sigma_{r}\right) \in\left(L^{*} \backslash\{\mathbf{0}\}\right)^{r}$ Gunnells and Sczech define the Dedekind sum

$$
D(L, \sigma, e, v):=\frac{1}{(2 \pi \sqrt{-1})^{\mathrm{wt}(e)}} \sum_{x \in L}^{\prime} \frac{\exp (2 \pi \sqrt{-1}\langle x, v\rangle)}{\left\langle x, \sigma_{1}\right\rangle^{e_{1}} \cdots\left\langle x, \sigma_{r}\right\rangle^{e_{r}}},
$$

where $\langle\rangle:, L \times L^{*} \rightarrow \mathbb{Z}$ is the pairing and $\sum^{\prime}$ means the terms with vanishing denominator are to be omitted. When $L=\mathbb{Z}^{\ell}$ we represent each $v \in L^{*} \otimes \mathbb{R}$ by a vector in $\mathbb{R}^{\ell}$ so that $\left\langle\left(m_{1}, \ldots, m_{\ell}\right),\left(v_{1}, \ldots, v_{\ell}\right)\right\rangle=m_{1} v_{1}+\cdots+m_{\ell} v_{\ell}$.

Let $\ell$ be the rank of the semisimple Lie algebra $\mathfrak{g}, r=\left|\Delta_{+}\right|$, and $W$ its Weyl group. Define an $\ell \times r$ integral matrix $\sigma(\mathfrak{g})$ whose $j$-th column $v_{j}$ provides the coefficients of $\alpha_{j} \in \Delta_{+}$in terms of the fundamental roots in $\Delta$. Let $e=(2 m, \ldots, 2 m) \in \mathbb{N}^{r}$. Then by [1, Prop. 8.4] we have

$$
\zeta_{W}(2 m, \mathfrak{g})=(2 \pi \sqrt{-1})^{2 m r} \frac{M(\mathfrak{g})^{2 m}}{|W|} D\left(\mathbb{Z}^{\ell}, \sigma(\mathfrak{g}), e, \mathbf{0}\right) .
$$

In 11 Gunnells and Sczech demonstrated how one can use the reciprocity law of higher-dimensional Dedekind sums to derived the Witten volume formulas of some Lie algebras. We are going to replace this tool by the following simple combinatorial lemma (see [7, p. 48]).

Lemma 2.1. Let $s, t$ be two positive integers. Let $x$ and $y$ be two non-zero real numbers such that $x+y \neq 0$. Then

$$
\frac{1}{x^{s} y^{t}}=\sum_{a=0}^{s-1}\left(\begin{array}{c}
t+a-1 \\
a
\end{array}\right) \frac{1}{x^{s-a}(x+y)^{t+a}}+\sum_{b=0}^{t-1}\left(\begin{array}{c}
s+b-1 \\
b
\end{array}\right) \frac{1}{y^{t-b}(x+y)^{s+b}} .
$$

To demonstrate this idea we have the following key lemma to be used many times later in the paper. Given any $\ell \times r$ matrix $\sigma=\left(\sigma_{1}, \ldots, \sigma_{r}\right)$ we denote $\left(\left(\sigma_{1}\right)_{e_{1}}, \ldots,\left(\sigma_{r}\right)_{e_{r}}\right)$ the new matrix obtained by repeating each linear form $\sigma_{j}$ exactly $e_{j}$ times, $j=1, \ldots, r$. For simplicity we further set

$$
Z\left(\left(\sigma_{1}\right)_{e_{1}}, \ldots,\left(\sigma_{r}\right)_{e_{r}}\right)=(2 \pi \sqrt{-1})^{e_{1}+\cdots+e_{r}} D\left(\mathbb{Z}^{\ell},\left(\sigma_{1}, \ldots, \sigma_{r}\right),\left(e_{1}, \ldots, e_{r}\right), \mathbf{0}\right)
$$


For example if

$$
M=\left(\begin{array}{ll}
1 & 1 \\
0_{4} & 2_{2}
\end{array}\right)=\left(\begin{array}{llllll}
1 & 1 & 1 & 1 & 1 & 1 \\
0 & 0 & 0 & 0 & 2 & 2
\end{array}\right)
$$

then

$$
Z(M)=-(2 \pi)^{6} D\left(\mathbb{Z}^{2},\left(\begin{array}{ll}
1 & 1 \\
0 & 2
\end{array}\right),(4,2), \mathbf{0}\right)
$$

Lemma 2.2. Suppose $a, b, c, d, e, f \in \mathbb{Z}$ such that $\operatorname{gcd}(a, b)=\operatorname{gcd}(c, d)=\operatorname{gcd}(e, f)=1$ and $u \lambda(a, b)+\lambda(c, d)=(e, f)$ for some nonzero constant $\lambda$ and $|u|=1,2$ or $1 / 2$. Let $\delta=\left|\begin{array}{ll}a & e \\ b & f\end{array}\right|$. If $|\delta|=1,2$, and $|u \delta|=1,2$ then for all positive integers $i, j$ and $k$ such that $w=i+j+k$ is even we have

$$
\begin{aligned}
Z\left(\begin{array}{ccc}
a & c & e \\
b_{i} & d_{j} & f_{k}
\end{array}\right) & =(2 \pi \sqrt{-1})^{w} \sum_{l=0}^{i}\left(\begin{array}{c}
i+j-l-1 \\
j-1
\end{array}\right) u^{i-l} \lambda^{i+j-l} \frac{B_{l} B_{w-l}}{l !(w-l) !} \alpha_{l, w}(\delta) \\
& +(2 \pi \sqrt{-1})^{w} \sum_{l=0}^{j}\left(\begin{array}{c}
i+j-l-1 \\
i-1
\end{array}\right) u^{i} \lambda^{i+j-l} \frac{B_{l} B_{w-l}}{l !(w-l) !} \alpha_{l, w}(u \delta),
\end{aligned}
$$

where $\alpha_{l, w}( \pm 1)=1$ and $\alpha_{l, w}( \pm 2)=1-1 / 2^{l}-1 / 2^{w-l}+2 / 2^{w}$.

Proof. Clearly we have

$$
\left|\begin{array}{ll}
a & e \\
b & f
\end{array}\right|=\delta \quad \Longrightarrow \quad\left|\begin{array}{ll}
c & e \\
d & f
\end{array}\right|=-u \delta
$$

For all integers $x$ and $y$ let $(x, y)^{\perp}=\left\{\left(m_{1}, m_{2}\right) \in \mathbb{Z}^{2}: x m_{1}+y m_{2}=0\right\}$. By the combinatorial Lemma 2.1 we have

$$
\begin{aligned}
Z\left(\begin{array}{lll}
a & c & e \\
b_{i} & d_{j} & f_{k}
\end{array}\right) & =\sum_{l=1}^{i}\left(\begin{array}{c}
i+j-l-1 \\
j-1
\end{array}\right) u^{i-l} \lambda^{i+j-l}\left[Z\left(\begin{array}{ll}
a & e \\
b_{l} & f_{w-l}
\end{array}\right)-Z_{(c, d)}\left(\begin{array}{ll}
a & e \\
b_{l} & f_{w-l}
\end{array}\right)\right] \\
& +\sum_{l=1}^{j}\left(\begin{array}{c}
i+j-l-1 \\
i-1
\end{array}\right) u^{i} \lambda^{i+j-l}\left[Z\left(\begin{array}{ll}
c & e \\
d_{l} & f_{w-l}
\end{array}\right)-Z_{(a, b)}\left(\begin{array}{ll}
c & e \\
d_{l} & f_{w-l}
\end{array}\right)\right] .
\end{aligned}
$$

Here for any sub lattice $L$ of $\mathbb{Z}^{2}$ the sum $Z_{L}$ is the sum $Z$ restricted to $L$. These terms in fact exactly correspond to those appearing on the right hand of the reciprocity law [1, (15)]. The condition $\operatorname{gcd}(c, d)=1$ implies that

$$
\begin{aligned}
Z_{(c, d) \perp}\left(\begin{array}{ll}
a & e \\
b_{l} & f_{w-l}
\end{array}\right) & =\sum_{\substack{m_{1}, m_{2} \in \mathbb{Z}, c m_{1}+d m_{2}=0 \\
a m_{1}+b m_{2} \neq 0, e m_{1}+f m_{2} \neq 0}} \frac{1}{\left(a m_{1}+b m_{2}\right)^{l}\left(e m_{1}+f m_{2}\right)^{w-l}} \\
& =\sum_{N \in \mathbb{Z}^{*}, m_{1}=d N, m_{2}=-c N} \frac{u^{l} \lambda^{l}}{\left(u \lambda a m_{1}+u \lambda b m_{2}\right)^{l}\left(e m_{1}+f m_{2}\right)^{w-l}} \\
& =\sum_{N \in \mathbb{Z}^{*}} \frac{u^{l} \lambda^{l}}{((e-\lambda c) d N-(f-\lambda d) c N)^{l}(e d N-f c N)^{w-l}}=\frac{1}{(u \delta)^{w}} \sum_{N \in \mathbb{Z}^{*}} \frac{u^{l} \lambda^{l}}{N^{w}}
\end{aligned}
$$


by (2.4) since $w$ is even. Hence by an easy binomial identity we get

$$
\sum_{l=0}^{i}\left(\begin{array}{c}
i+j-l-1 \\
j-1
\end{array}\right) u^{i-l} \lambda^{i+j-l} Z_{(c, d)^{\perp}}\left(\begin{array}{ll}
a & e \\
b_{l} & f_{w-l}
\end{array}\right)=\left(\begin{array}{c}
i+j-1 \\
j
\end{array}\right) \frac{u^{i} \lambda^{i+j}}{(u \delta)^{w}} \sum_{N \in \mathbb{Z}^{*}} \frac{1}{N^{w}} .
$$

Notice that for all integral matrix $\sigma=\left(\begin{array}{cc}x & y \\ s & t\end{array}\right)$ of determinant $\delta$ and positive integers $j, k$ with $j+k \geq 4$ we have by [1, (4)]

$$
Z\left(\begin{array}{ll}
x & y \\
s_{j} & t_{k}
\end{array}\right)=(2 \pi \sqrt{-1})^{j+k} D\left(\mathbb{Z}^{2}, \sigma,(j, k), \mathbf{0}\right)=\frac{(2 \pi \sqrt{-1})^{j+k}}{j ! k !|\delta|} \sum_{z \in \mathbb{Z}^{2} / \sigma \mathbb{Z}^{2}} \mathcal{B}_{j}\left(\sigma^{-1} z\right) \mathcal{B}_{k}\left(\sigma^{-1} z\right),
$$

where $\mathcal{B}_{j}(x)$ are the Bernoulli polynomials. When $u \delta= \pm 1$ the quantity in (2.6) provides exactly the $l=0$ term in the sum of (2.3) by the following formula known to Euler: for even positive integer $w$

$$
\sum_{N \in \mathbb{Z}^{*}} \frac{1}{N^{w}}=2 \zeta(w)=-(2 \pi)^{w} \frac{B_{w}}{w !}
$$

When $u \delta= \pm 2$ by the assumption $\operatorname{gcd}(c, d)=\operatorname{gcd}(e, f)=1$ and (2.7) we get

$$
Z\left(\begin{array}{ll}
c & e \\
d_{l} & f_{w-l}
\end{array}\right)=\frac{(2 \pi \sqrt{-1})^{w}}{2} \frac{B_{l} B_{w-l}}{l !(w-l) !}\left(1+\left(\frac{2}{2^{l}}-1\right)\left(\frac{2}{2^{w-l}}-1\right)\right) .
$$

When $l=0$ we find that (2.8) is equal to $B_{w} /\left(2^{w} w !\right)$ and therefore (2.6) again provides exactly the $l=0$ term in the sum of (2.3) .

Similarly, the $l=0$ term in the sum of (2.2) can be obtained by the sum of $Z_{(a, b)^{\perp}}\left(\begin{array}{lll}c & e \\ d_{l} & f_{w-l}\end{array}\right)$ in (2.5). This finishes the proof of the lemma.

We can obtain (1.1) immediately by applying the lemma to

$$
Z\left(\begin{array}{lll}
1 & 0 & 1 \\
0_{2 m} & 1_{2 m} & 1_{2 m}
\end{array}\right)
$$

To aid our computation we represent the procedure in Lemma 2.2 by the following picture: the left is self-evident while the right is more elegant by only recording the merged columns. For example, in $1 / x y(x+y)=1 / x(x+y)^{2}+1 / y(x+y)^{2}$ we may think $x, y$ and $x+y$ correspond to the first three columns and $1 / x(x+y)^{2}$ is said to be obtained by merge the 2 nd column to the $3 \mathrm{rd}$. We can generalize Lemma 2.1 and apply it to any three linearly dependent columns of a general matrix. In the following picture, a circled column number between any two sub-nodes signifies the column to which the two nodes are merged. We call the tree in the right picture a computation tree.

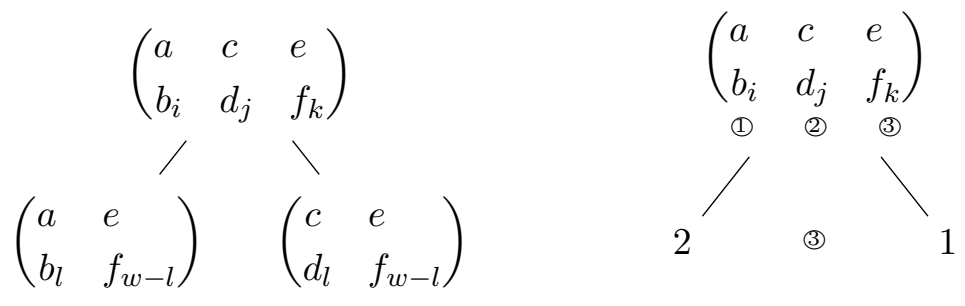

We have the following partial generalization of Lemma 2.2 whose proof is left to the interested reader. 
Lemma 2.3. Let $\sigma=\left(\sigma_{1}, \ldots, \sigma_{r}\right)$ be an $(r-1) \times r$ matrix with $r \geq 3$. Let $e_{1}, \ldots, e_{r} \in \mathbb{N}$ and put $s=e_{1}+e_{2}+e_{3}$. Suppose $u= \pm 1, u \lambda \sigma_{1}+\lambda \sigma_{2}=\sigma_{3}$ for some non-zero constant $\lambda$ and $\operatorname{det}\left(\sigma_{2}, \ldots, \sigma_{r}\right)= \pm 1$. If both $\sigma_{1}$ and $\sigma_{2}$ have at least one component equal to \pm 1 then

$$
\begin{array}{r}
Z\left(\left(\sigma_{1}\right)_{e_{1}}, \ldots,\left(\sigma_{r}\right)_{e_{r}}\right)=(2 \pi \sqrt{-1})^{w} \frac{B_{e_{4}} \ldots B_{e_{r}}}{e_{4} ! \ldots e_{r} !} u^{e_{1}} \lambda^{e_{1}+e_{2}}\left\{\sum_{l=0}^{e_{1}}\left(\begin{array}{c}
e_{1}+e_{2}-l-1 \\
e_{2}-1
\end{array}\right) \frac{u^{l} B_{l} B_{s-l}}{\lambda^{l} l !(s-l) !}\right. \\
\left.+\sum_{l=0}^{e_{2}}\left(\begin{array}{c}
e_{1}+e_{2}-l-1 \\
e_{1}-1
\end{array}\right) \frac{B_{l} B_{s-l}}{\lambda^{l} l !(s-l) !}\right\} .
\end{array}
$$

Remark. It is incorrect to generalize Lemma 2.2 to arbitrary rank without the assumption that $\sigma_{1}$ and $\sigma_{2}$ have at least one component equal to \pm 1 .

Definition. If a node of binary tree has the following property then we say it's a good parent: either it is not a grandparent, or every one of its descendants names one of their children the same as its only sibling.

Proposition 2.1. Let $\sigma=\left(\sigma_{1}, \ldots, \sigma_{r}\right)$ be an $\ell \times r$ matrix with $r \geq \ell+1 \geq 2$. Suppose that every column has some component equal to \pm 1 . Let $e_{1}, \ldots, e_{r} \in \mathbb{N}$ and put $s=e_{1}+e_{2}+e_{3}$. Assume $\sigma$ is a good parent in its computation binary tree. Further assume that we have the top part of the computation binary tree as follows if it has four grandchildren:

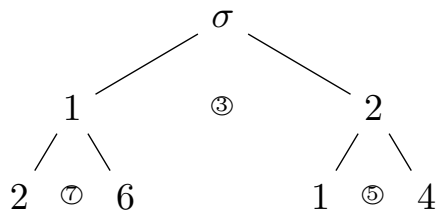

Here, 3,5, and 7 may or may not be the same but $5 \neq 1,2,4$ and $7 \neq 1,2,6$. Suppose every node in the second to the last generation satisfies the conditions in Lemma 2.2 (resp. Lemma 2.3) if it has rank two (resp. greater than two). If $\lambda_{1} \sigma_{1}+\lambda_{2} \sigma_{2}=\sigma_{3}$. Then

$$
\begin{aligned}
Z\left(\left(\sigma_{1}\right)_{e_{2}}, \ldots,\left(\sigma_{r}\right)_{e_{r}}\right) & =\lambda_{1}^{e_{1}} \lambda_{2}^{e_{2}} \sum_{i=0}^{e_{2}}\left(\begin{array}{c}
e_{1}+e_{2}-i-1 \\
e_{1}-1
\end{array}\right) \frac{1}{\lambda_{2}^{i}} Z\left(\left(\sigma_{2}\right)_{i},\left(\sigma_{3}\right)_{s-i},\left(\sigma_{4}\right)_{e_{4}}, \ldots,\left(\sigma_{r}\right)_{e_{r}}\right) \\
& +\lambda_{1}^{e_{1}} \lambda_{2}^{e_{2}} \sum_{i=0}^{e_{1}}\left(\begin{array}{c}
e_{1}+e_{2}-i-1 \\
e_{2}-1
\end{array}\right) \frac{1}{\lambda_{1}^{i}} Z\left(\left(\sigma_{1}\right)_{i},\left(\sigma_{3}\right)_{s-i},\left(\sigma_{4}\right)_{e_{4}}, \ldots,\left(\sigma_{r}\right)_{e_{r}}\right) .
\end{aligned}
$$

Proof. When $r=\ell+1$ the proposition follows from Lemma 2.2 and Lemma 2.3) by our assumption since now the second to the last generation is exactly $\sigma$ itself. Assume $r>\ell+1$. By Lemma 2.1 it's clear that

$$
\begin{aligned}
& Z\left(\left(\sigma_{1}\right)_{e_{1}}, \ldots,\left(\sigma_{r}\right)_{e_{r}}\right) \\
= & \lambda_{1}^{e_{1}} \lambda_{2}^{e_{2}} \sum_{i=1}^{e_{2}}\left(\begin{array}{c}
e_{1}+e_{2}-i-1 \\
e_{1}-1
\end{array}\right) \frac{1}{\lambda_{2}^{i}}\left[Z\left(\left(\sigma_{2}\right)_{i},\left(\sigma_{3}\right)_{s-i},\left(\sigma_{4}\right)_{e_{4}}, \ldots,\left(\sigma_{r}\right)_{e_{r}}\right)-Z_{\sigma_{1}^{\perp}}\right] \\
+ & \lambda_{1}^{e_{1}} \lambda_{2}^{e_{2}} \sum_{i=1}^{e_{1}}\left(\begin{array}{c}
e_{1}+e_{2}-i-1 \\
e_{2}-1
\end{array}\right) \frac{1}{\lambda_{1}^{i}}\left[Z\left(\left(\sigma_{1}\right)_{i},\left(\sigma_{3}\right)_{s-i},\left(\sigma_{4}\right)_{e_{4}}, \ldots,\left(\sigma_{r}\right)_{e_{r}}\right)-Z_{\sigma_{2}^{\perp}}\right] .
\end{aligned}
$$


With fixed $\ell$ we now use induction on $r$ to show that

$$
\begin{aligned}
& \lambda_{2}^{-i} Z_{\sigma_{1}^{\perp}}\left(\left(\sigma_{3}\right)_{s},\left(\sigma_{4}\right)_{e_{4}}, \ldots,\left(\sigma_{r}\right)_{e_{r}}\right)=\left.Z\left(\left(\sigma_{1}\right)_{i},\left(\sigma_{3}\right)_{s-i},\left(\sigma_{4}\right)_{e_{4}}, \ldots,\left(\sigma_{r}\right)_{e_{r}}\right)\right|_{i=0}, \\
& \lambda_{1}^{-i} Z_{\sigma_{2}^{\perp}}\left(\left(\sigma_{3}\right)_{s},\left(\sigma_{4}\right)_{e_{4}}, \ldots,\left(\sigma_{r}\right)_{e_{r}}\right)=\left.Z\left(\left(\sigma_{2}\right)_{i},\left(\sigma_{3}\right)_{s-i},\left(\sigma_{4}\right)_{e_{4}}, \ldots,\left(\sigma_{r}\right)_{e_{r}}\right)\right|_{i=0},
\end{aligned}
$$

which yields the Lemma immediately by the simple combinatorial identities

$$
\sum_{i=1}^{e_{1}}\left(\begin{array}{c}
e_{1}+e_{2}-i-1 \\
e_{2}-1
\end{array}\right)=\left(\begin{array}{c}
e_{1}+e_{2}-1 \\
e_{2}
\end{array}\right), \quad \sum_{i=1}^{e_{2}}\left(\begin{array}{c}
e_{1}+e_{2}-i-1 \\
e_{1}-1
\end{array}\right)=\left(\begin{array}{c}
e_{1}+e_{2}-1 \\
e_{1}
\end{array}\right) .
$$

By the computation tree we may assume $\mu_{1} \sigma_{1}+\mu_{2} \sigma_{4}=\sigma_{5}$. Notice that for any $x \in \sigma_{1}^{\perp}$ we have

$$
\begin{aligned}
& \lambda_{2}\left\langle\sigma_{2}, x\right\rangle=\lambda_{1}\left\langle\sigma_{1}, x\right\rangle+\lambda_{2}\left\langle\sigma_{2}, x\right\rangle=\left\langle\sigma_{3}, x\right\rangle, \\
& \mu_{2}\left\langle\sigma_{4}, x\right\rangle=\mu_{1}\left\langle\sigma_{1}, x\right\rangle+\mu_{2}\left\langle\sigma_{4}, x\right\rangle=\left\langle\sigma_{5}, x\right\rangle . H
\end{aligned}
$$

ence (if (3) $=$ (5) then $e_{5}=0$ )

$$
\begin{aligned}
\lambda_{2}^{-i} Z_{\sigma_{1}^{\perp}}\left(\left(\sigma_{2}\right)_{i},\left(\sigma_{3}\right)_{s-i},\left(\sigma_{4}\right)_{e_{4}}, \ldots,\left(\sigma_{r}\right)_{e_{r}}\right) & =Z_{\sigma_{1}^{\perp}}\left(\left(\sigma_{3}\right)_{s},\left(\sigma_{4}\right)_{e_{4}}, \ldots,\left(\sigma_{r}\right)_{e_{r}}\right) \\
& =\mu_{2}^{e_{4}} Z_{\sigma_{1}^{\perp}} Z\left(\left(\sigma_{3}\right)_{s},\left(\sigma_{5}\right)_{e_{4}+e_{5}},\left(\sigma_{6}\right)_{e_{6}}, \ldots,\left(\sigma_{r}\right)_{e_{r}}\right) .
\end{aligned}
$$

On the other hand, by induction assumption we get

$$
\begin{aligned}
& Z\left(\left(\sigma_{1}\right)_{i},\left(\sigma_{3}\right)_{s-i},\left(\sigma_{4}\right)_{e_{4}}, \ldots,\left(\sigma_{r}\right)_{e_{r}}\right) \\
= & \mu_{1}^{i} \mu_{2}^{e_{4}} \sum_{j=0}^{e_{4}}\left(\begin{array}{c}
e_{4}+i-j-1 \\
i-1
\end{array}\right) \frac{1}{\mu_{2}^{j}} Z\left(\left(\sigma_{3}\right)_{s-i},\left(\sigma_{4}\right)_{j},\left(\sigma_{5}\right)_{e_{4}+e_{5}+i-j},\left(\sigma_{6}\right)_{e_{6}}, \ldots,\left(\sigma_{r}\right)_{e_{r}}\right) \\
+ & \mu_{1}^{i} \mu_{2}^{e_{4}} \sum_{j=0}^{i}\left(\begin{array}{c}
e_{4}+i-j-1 \\
e_{4}-1
\end{array}\right) \frac{1}{\mu_{1}^{j}} Z\left(\left(\sigma_{1}\right)_{j},\left(\sigma_{3}\right)_{s-i},\left(\sigma_{5}\right)_{e_{4}+e_{5}+i-j},\left(\sigma_{6}\right)_{e_{6}}, \ldots,\left(\sigma_{r}\right)_{e_{r}}\right) .
\end{aligned}
$$

Taking $i=0$ in this expression we see that the first sum is vacuous because of the binomial coefficient while the second sum is reduced to just one term:

$$
\begin{aligned}
\mu_{1}^{i-j} \mu_{2}^{e_{4}} Z\left(\left(\sigma_{1}\right)_{j},\left(\sigma_{3}\right)_{s-i},\left(\sigma_{5}\right)_{e_{4}+e_{5}+i-j},\left(\sigma_{6}\right)_{e_{6}}, \ldots,\right. & \left.\left(\sigma_{r}\right)_{e_{r}}\right)\left.\right|_{i=j=0} \\
& =\mu_{2}^{e_{4}} Z_{\sigma_{1}^{\perp}}\left(\left(\sigma_{3}\right)_{s},\left(\sigma_{5}\right)_{e_{4}+e_{5}},\left(\sigma_{6}\right)_{e_{6}}, \ldots,\left(\sigma_{r}\right)_{e_{r}}\right),
\end{aligned}
$$

by induction assumption. Thus equation (2.11) follows from (2.12). The proof of (2.11) is exactly the same. This concludes the proof of the proposition.

\section{The $\mathfrak{s o}(5)$ CASE}

Let $m \in \mathbb{N}$ and $n=2 m$. By the above we can write

$$
\zeta_{\mathfrak{s o}(5)}(n, n, n, n)=\sum_{a, b=1}^{\infty} \frac{1}{a^{n} b^{n}(a+b)^{n}(a+2 b)^{n}}=\frac{(2 \pi)^{8 m}}{8} D\left(\mathbb{Z}^{2}, \sigma,(1, \ldots, 1), \mathbf{0}\right)
$$

where $(1, \ldots, 1) \in \mathbb{N}^{4 n}$, the matrix $\sigma=\sigma(n, n, n, n)$ and

$$
\sigma(a, b, c, d)=\left(\begin{array}{llll}
1 & 0 & 1 & 1 \\
0_{a} & 1_{b} & 1_{c} & 2_{d}
\end{array}\right)
$$


To prepare for the $\mathfrak{s o}(7)$ case we first prove a generalization of the $\mathfrak{s o}(5)$ case by the following computation tree

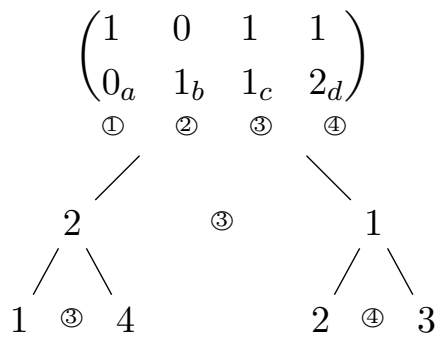

Theorem 3.1. Let $a, b, c, d \in \mathbb{N}$ and suppose at most one of them is 1 . Put $\beta_{j, w}=B_{j} B_{w-j} /(j !(w-$ $j)$ !). If $w=a+b+c+d$ is even then

$$
\begin{aligned}
Z\left(\begin{array}{cccc}
1 & 0 & 1 & 1 \\
0_{a} & 1_{b} & 1_{c} & 2_{d}
\end{array}\right) & =\sum_{i=0}^{b}\left(\begin{array}{c}
a+b-i-1 \\
a-1
\end{array}\right)\left(\sum_{j=0}^{i}\left(\begin{array}{c}
w-d-j-1 \\
w-d-i-1
\end{array}\right) \beta_{j, w}+\sum_{j=0}^{w-d-i}\left(\begin{array}{c}
w-d-j-1 \\
i-1
\end{array}\right) \beta_{j, w}\right) \\
& +\sum_{i=0}^{a}\left(\begin{array}{c}
a+b-i-1 \\
b-1
\end{array}\right)\left(\sum_{j=0}^{d}\left(\begin{array}{c}
d+i-j-1 \\
i-1
\end{array}\right) \frac{\beta_{j, w}}{2^{d+i-j}}+\sum_{j=0}^{i}\left(\begin{array}{c}
d+i-j-1 \\
d-1
\end{array}\right) \frac{\beta_{j, w}}{2^{d+i-j}}\right)
\end{aligned}
$$

Proof. It's easy to check that all the $2 \times 2$ minors of $\left(\begin{array}{llll}1 & 0 & 1 & 1 \\ 0_{a} & 1_{b} & 1_{c} & 2_{d}\end{array}\right)$ have determinant \pm 1 or \pm 2 . So we can apply Proposition 2.1 and get

$$
\begin{aligned}
& Z\left(\begin{array}{llll}
1 & 0 & 1 & 1 \\
0_{a} & 1_{b} & 1_{c} & 2_{d}
\end{array}\right) \\
= & \sum_{i=0}^{b}\left(\begin{array}{c}
a+b-i-1 \\
a-1
\end{array}\right) Z\left(\begin{array}{lll}
0 & 1 & 1 \\
1_{i} & 1_{w-d-i} & 2_{d}
\end{array}\right)+\sum_{i=0}^{a}\left(\begin{array}{c}
a+b-i-1 \\
b-1
\end{array}\right) Z\left(\begin{array}{lll}
1 & 1 & 1 \\
0_{i} & 1_{w-d-i} & 2_{d}
\end{array}\right) .
\end{aligned}
$$

The theorem now follows from Lemma 2.2 directly.

This implies the following as an immediate corollary. In the rest of the paper for nonnegative integer $l$ and even integer $w \geq 4$ we set $\beta_{l, w}=(2 \pi)^{w} B_{l} B_{w-l} /(w-l) ! / l !$.

Theorem 3.2. Let $n$ be a positive even integer and $w=4 n$. Set $\left(\begin{array}{c}t \\ -1\end{array}\right)=0$ for all $t$. Then

$$
\begin{aligned}
\frac{8 \zeta_{W}(n, \mathfrak{s o}(5))}{6^{n}(2 \pi)^{4 n}} & =\sum_{i=0}^{n}\left(\begin{array}{c}
2 n-i-1 \\
n-1
\end{array}\right)\left[\sum_{j=0}^{3 n-i}\left(\begin{array}{c}
3 n-j-1 \\
i-1
\end{array}\right) \beta_{j, w}+\sum_{j=0}^{i}\left(\begin{array}{c}
3 n-j-1 \\
3 n-i-1
\end{array}\right) \beta_{j, w}\right. \\
& \left.+\sum_{j=0}^{n} \frac{1}{2^{n+i-j}}\left(\begin{array}{c}
n+i-j-1 \\
i-1
\end{array}\right) \beta_{j, w}+\sum_{j=0}^{i} \frac{1}{2^{n+i-j}}\left(\begin{array}{c}
n+i-j-1 \\
n-1
\end{array}\right) \beta_{j, w}\right] .
\end{aligned}
$$

Proof. Clear.

Remark. By exchange the order of summation in the theorem we see that our formula agrees with that of Matsumoto et al. in [5]. 


\section{THE $\mathfrak{g}_{2}$ CASE}

By definition we have $\zeta_{W}\left(s ; \mathfrak{g}_{2}\right)=120^{s} \zeta_{\mathfrak{g}_{2}}(s, \ldots, s)$ where

$$
\zeta_{\mathfrak{g}_{2}}\left(s_{1}, \ldots, s_{6}\right)=\sum_{a, b=1}^{\infty} \frac{1}{a^{s_{1}} b^{s_{2}}(a+b)^{s_{3}}(a+2 b)^{s_{4}}(a+3 b)^{s_{5}}(2 a+3 b)^{s_{6}}} .
$$

In the rest of this section we fix a positive even integer $n=2 m$. By (2.1) we have

$$
\zeta_{W}\left(2 m, \mathfrak{g}_{2}\right)=\frac{(2 \pi)^{12 m} 120^{2 m}}{12} D\left(\mathbb{Z}^{2}, \sigma,(1, \ldots, 1), \mathbf{0}\right)
$$

where $(1, \ldots, 1) \in \mathbb{N}^{6 n}$ and

$$
\sigma=\left(\begin{array}{llllll}
1 & 0 & 1 & 1 & 1 & 2 \\
0_{n} & 1_{n} & 1_{n} & 2_{n} & 3_{n} & 3_{n}
\end{array}\right)
$$

Similar to the case of $\mathfrak{s o}(5)$ we can proceed using the following picture:

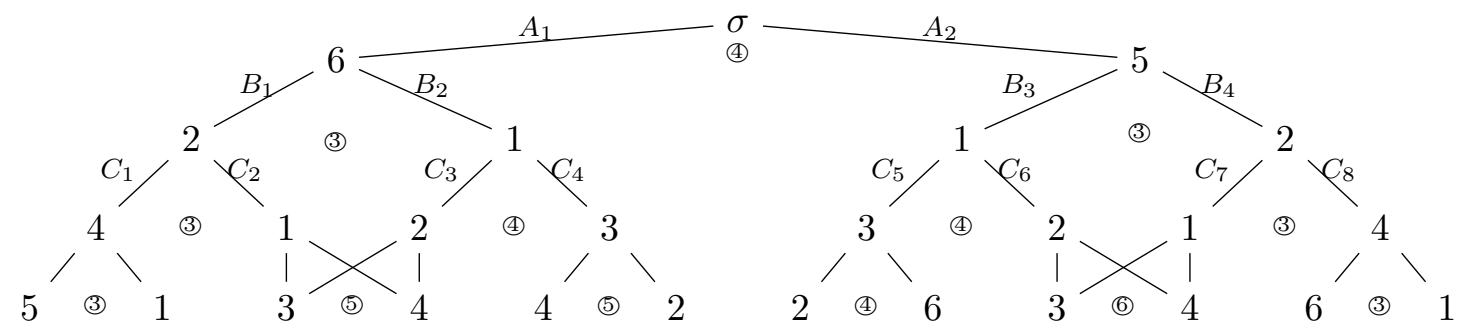

Set $\delta_{2}(l)=1+2^{1-6 n}-2^{-l}-2^{l-6 n}, \beta_{l, w}=(2 \pi)^{w} B_{l} B_{w-l} /(w-l) ! / l !$ and $\beta_{l, w}^{\prime \prime}=\delta_{2}(l) \beta_{l, w}$. We thus get

$$
Z\left(\begin{array}{cccccc}
1 & 0 & 1 & 1 & 1 & 2 \\
0_{n} & 1_{n} & 1_{n} & 2_{n} & 3_{n} & 3_{n}
\end{array}\right)=\sum_{i=0}^{n}\left(\begin{array}{c}
2 n-i-1 \\
n-1
\end{array}\right) \frac{A_{1}(i)+A_{2}(i)}{3^{2 n-i}}
$$

where

$$
A_{1}(i)=Z\left(\begin{array}{lllll}
1 & 0 & 1 & 1 & 1 \\
0_{n} & 1_{n} & 1_{n} & 2_{3 n-i} & 3_{i}
\end{array}\right), \quad A_{1}(i)=Z\left(\begin{array}{lllll}
1 & 0 & 1 & 1 & 1 \\
0_{n} & 1_{n} & 1_{n} & 2_{3 n-i} & 3_{i}
\end{array}\right) .
$$

Note that $\sigma$ is not a good parent so we have to check the two perpendicular terms can indeed be absorbed into the summand when setting $i=0$ in (4.2). This is not too difficult after finding out the explicit expressions of $A_{1}$ and $A_{2}$ both of which are good parents. Therefore it follows from Proposition 2.1 that

$$
\begin{aligned}
& A_{1}(i)=\sum_{j=0}^{n}\left(\begin{array}{c}
2 n-j-1 \\
n-1
\end{array}\right)\left(B_{1}(i, j)+B_{2}(i, j)\right), \\
& A_{2}(i)=\sum_{j=0}^{n}\left(\begin{array}{c}
2 n-j-1 \\
n-1
\end{array}\right)\left(B_{3}(i, j)+B_{4}(i, j)\right),
\end{aligned}
$$


where

$$
\begin{aligned}
B_{1}(i, j)=Z\left(\begin{array}{llll}
1 & 1 & 1 & 1 \\
0_{j} & 1_{3 n-j} & 2_{3 n-i} & 3_{i}
\end{array}\right)= & \sum_{k=0}^{j}\left(\begin{array}{c}
3 n-i+j-k-1 \\
3 n-i-1
\end{array}\right) \frac{C_{1}(i, k)}{2^{3 n-i+j-k}} \\
& +\sum_{k=0}^{3 n-i}\left(\begin{array}{c}
3 n-i+j-k-1 \\
j-1
\end{array}\right) \frac{C_{2}(i, k)}{2^{3 n-i+j-k},} \\
B_{2}(i, j)=Z\left(\begin{array}{llll}
0 & 1 & 1 & 1 \\
1_{j} & 1_{3 n-j} & 2_{3 n-i} & 3_{i}
\end{array}\right)= & \sum_{k=0}^{3 n-j}\left(\begin{array}{c}
3 n-k-1 \\
j-1
\end{array}\right) C_{3}(i, k)+\sum_{k=0}^{j}\left(\begin{array}{c}
3 n-k-1 \\
3 n-j-1
\end{array}\right) C_{4}(i, k), \\
B_{3}(i, j)=Z\left(\begin{array}{llll}
0 & 1 & 1 & 2 \\
1_{j} & 1_{3 n-j} & 2_{3 n-i} & 3_{i}
\end{array}\right)= & \sum_{k=0}^{j}\left(\begin{array}{c}
3 n-k-1 \\
3 n-j-1
\end{array}\right) C_{3}(i, k)+\sum_{k=0}^{3 n-j}\left(\begin{array}{c}
3 n-k-1 \\
j-1
\end{array}\right) C_{4}(i, k), \\
B_{4}(i, j)=Z\left(\begin{array}{llll}
1 & 1 & 1 & 2 \\
0_{j} & 1_{3 n-j} & 2_{3 n-i} & 3_{i}
\end{array}\right)= & \sum_{k=0}^{3 n-i}\left(\begin{array}{c}
3 n-i+j-k-1 \\
j-1
\end{array}\right) \frac{C_{4}(i, k)}{2^{3 n-i+j-k}} \\
& +\sum_{k=0}^{j}\left(\begin{array}{c}
3 n-i+j-k-1 \\
3 n-i-1
\end{array}\right) \frac{C_{8}(i, k)}{2^{3 n-i+j-k},},
\end{aligned}
$$

where, by using Lemma 2.2 we have

$$
\begin{aligned}
& C_{1}(i, k)=Z\left(\begin{array}{lll}
1 & 1 & 1 \\
0_{k} & 1_{6 n-k-i} & 3_{i}
\end{array}\right)=\sum_{l=0}^{i}\left(\begin{array}{c}
i+k-l-1 \\
k-1
\end{array}\right) \frac{2^{k} \delta_{2}(l)}{3^{i+k-l}} \beta_{l, w}+\sum_{l=0}^{k}\left(\begin{array}{c}
i+k-l-1 \\
i-1
\end{array}\right) \frac{2^{k-l}}{3^{i+k-l}} \beta_{l, w} \\
& C_{2}(i, k)=Z\left(\begin{array}{lll}
1 & 1 & 1 \\
1_{6 n-k-i} & 2_{k} & 3_{i}
\end{array}\right)=\sum_{l=0}^{i}\left(\begin{array}{c}
i+k-l-1 \\
k-1
\end{array}\right)(-1)^{i} 2^{k} \delta_{2}(l) \beta_{l, w}+\sum_{l=0}^{k}\left(\begin{array}{c}
i+k-l-1 \\
i-1
\end{array}\right)(-1)^{i} 2^{k-l} \beta_{l, w}, \\
& C_{3}(i, k)=Z\left(\begin{array}{lll}
1 & 1 & 1 \\
1_{k} & 2_{6 n-k-i} & 3_{i}
\end{array}\right)=\sum_{l=0}^{i}\left(\begin{array}{c}
i+k-l-1 \\
k-1
\end{array}\right)(-1)^{i} \frac{\beta_{l, w}}{2^{k+i-l}}+\sum_{l=0}^{k}\left(\begin{array}{c}
i+k-l-1 \\
i-1
\end{array}\right)(-1)^{i} \frac{\beta_{l, w}}{2^{k+i-l}}, \\
& C_{4}(i, k)=Z\left(\begin{array}{lll}
0 & 1 & 1 \\
1_{k} & 2_{6 n-k-i} & 3_{i}
\end{array}\right)=\sum_{l=0}^{k}\left(\begin{array}{c}
i+k-l-1 \\
i-1
\end{array}\right)(-1)^{k} \beta_{l, w}+\sum_{l=0}^{i}\left(\begin{array}{c}
i+k-l-1 \\
k-1
\end{array}\right)(-1)^{k} \beta_{l, w}, \\
& C_{8}(i, k)=Z\left(\begin{array}{ccc}
1 & 1 & 2 \\
0_{k} & 1_{6 n-k-i} & 3_{i}
\end{array}\right)=\sum_{l=0}^{k}\left(\begin{array}{c}
i+k-l-1 \\
i-1
\end{array}\right) \frac{\beta_{l, w}}{3^{k+i-l}}+\sum_{l=0}^{i}\left(\begin{array}{c}
i+k-l-1 \\
k-1
\end{array}\right) \frac{\beta_{l, w}}{3^{k+i-l}} .
\end{aligned}
$$

Putting everything together we finally arrive at

Theorem 4.1. Let $n$ be a positive even integer. Let $w=6 n$ and set $\left(\begin{array}{c}t \\ -1\end{array}\right)=0$ for all $t$. Write $\beta_{l, w}=(2 \pi)^{w} B_{l} B_{w-l} /(w-l) ! / l !$. Then

$$
\begin{aligned}
\frac{12 \zeta_{W}\left(n, \mathfrak{g}_{2}\right)}{120^{n}} & =\sum_{i=0}^{n}\left(\begin{array}{c}
2 n-i-1 \\
n-1
\end{array}\right) 3^{i-2 n} \sum_{j=0}^{n}\left(\begin{array}{c}
2 n-j-1 \\
n-1
\end{array}\right) \times \\
& \times\left\{\sum_{k=0}^{j}\left(\begin{array}{c}
3 n-i+j-k-1 \\
3 n-i-1
\end{array}\right) 2^{i+k-3 n-j} \sum_{t=1}^{2} \sum_{l=0}^{\lambda_{t}}\left(\begin{array}{c}
i+k-l-1 \\
i+k-\lambda_{t}-1
\end{array}\right) \frac{1+2^{k} \delta_{t}(l)}{3^{i+k-l}} \beta_{l, w}\right.
\end{aligned}
$$




$$
\begin{aligned}
& +\sum_{k=0}^{3 n-i}\left(\begin{array}{c}
3 n-i+j-k-1 \\
j-1
\end{array}\right) \sum_{t=1}^{2} \sum_{l=0}^{\lambda_{t}}\left(\begin{array}{c}
i+k-l-1 \\
i+k-\lambda-1
\end{array}\right)\left((-1)^{k}+(-1)^{i} 2^{k} \delta_{t}(l)\right) \beta_{l, w} \\
& +\sum_{k=0}^{3 n-j}\left(\begin{array}{c}
3 n-k-1 \\
j-1
\end{array}\right) \sum_{\lambda=i, k} \sum_{l=0}^{\lambda}\left(\begin{array}{c}
i+k-l-1 \\
i+k-\lambda-1
\end{array}\right)\left((-1)^{k}+(-1)^{i} 2^{l-i-k}\right) \beta_{l, w} \\
& \left.+\sum_{k=0}^{j}\left(\begin{array}{c}
3 n-k-1 \\
3 n-j-1
\end{array}\right) \sum_{\lambda=i, k} \sum_{l=0}^{\lambda}\left(\begin{array}{c}
i+k-l-1 \\
i+k-\lambda-1
\end{array}\right)\left((-1)^{k}+(-1)^{i} 2^{l-i-k}\right) \beta_{l, w}\right\},
\end{aligned}
$$

where $\lambda_{1}=k, \delta_{1}(l)=2^{-l}, \lambda_{2}=i$, and $\delta_{2}(l)=1+2^{1-6 n}-2^{-l}-2^{l-6 n}$.

Remark. Although we can not verify the agreement of our theorem with [4] we are sure their result will follow by choosing another computation tree. However, we find our data for $\zeta_{W}\left(2 m, \mathfrak{g}_{2}\right)$ $(m \leq 10)$ agree with those in [4]. We have also verified numerically the correctness of these values by using the definition (4.1).

\section{The CASEs of $\mathfrak{s o}(7)$ AND $\mathfrak{s p}(6)$}

By definition

$$
\zeta_{W}(n ; \mathfrak{s o}(7))=720^{n} \sum_{m_{1}, m_{2}, m_{3}=1}^{\infty}\left(\frac{1 /\left(m_{1} m_{2} m_{3}\left(m_{1}+m_{2}\right)\left(m_{2}+m_{3}\right)\left(2 m_{2}+m_{3}\right)\right)}{\left(2 m_{1}+2 m_{2}+m_{3}\right)\left(m_{1}+2 m_{2}+m_{3}\right)\left(m_{1}+m_{2}+m_{3}\right)}\right)^{n}
$$

and

$$
\zeta_{W}(n ; \mathfrak{s p}(6))=720^{n} \sum_{m_{1}, m_{2}, m_{3}=1}^{\infty}\left(\frac{1 /\left(m_{1} m_{2} m_{3}\left(m_{1}+m_{2}\right)\left(m_{2}+m_{3}\right)\left(m_{2}+2 m_{3}\right)\right)}{\left(m_{1}+m_{2}+m_{3}\right)\left(m_{1}+m_{2}+2 m_{3}\right)\left(m_{1}+2 m_{2}+2 m_{3}\right)}\right)^{n} .
$$

The corresponding matrix to $\mathfrak{s o}(7)$ is

$$
\sigma=\left(\begin{array}{lllllllll}
1 & 0 & 0 & 1 & 0 & 0 & 2 & 1 & 1 \\
0 & 1 & 0 & 1 & 1 & 2 & 2 & 2 & 1 \\
0_{n} & 0_{n} & 1_{n} & 0_{n} & 1_{n} & 1_{n} & 1_{n} & 1_{n} & 1_{n}
\end{array}\right) .
$$

Given four column vectors $\sigma_{1}, \ldots, \sigma_{4}$ let $S\left(\sigma_{1}, \ldots, \sigma_{4}\right)$ be the set of the four possible choices of three columns. Then every triple columns of the following are linearly dependent:

$$
\{(1,5,9),(4,5,8)\} \cup S(1,6,7,8) \cup S(2,3,5,6) \cup S(3,4,7,9) .
$$

These fourteen dependencies are the only 3-column dependencies and will be used critically in the computation tree of $\zeta_{W}(n, \mathfrak{s o}(7))$ for even positive integer $n$. If we consider the matrix corresponding to $\mathfrak{s p}(6)$

$$
\left(\begin{array}{ccccccccc}
1 & 0 & 0 & 1 & 0 & 0 & 1 & 1 & 1 \\
0 & 1 & 0 & 1 & 1 & 1 & 2 & 1 & 1 \\
0_{n} & 0_{n} & 1_{n} & 0_{n} & 1_{n} & 2_{n} & 2_{n} & 2_{n} & 1_{n} \\
\text { (1) } & \text { (2) } & \text { (3) } & \text { (4) } & \text { (5) } & \text { (6) } & \text { (1) } & \text { (8) } & \text { (9) }
\end{array}\right)
$$

we find the following similar combinations of columns:

$$
\{(2,7,8),(4,6,7)\} \cup S(2,3,5,6) \cup S(1,5,7,9) \cup S(3,4,8,9) .
$$


But these can be obtained exactly from (5.2) by the permutation (12)(398657).

We may construct the following computation tree for $\mathfrak{s o}(7)$ as in the previous cases. A similar computation tree for $\mathfrak{s p}(6)$ can then be obtained by applying the permutation (12)(398657).

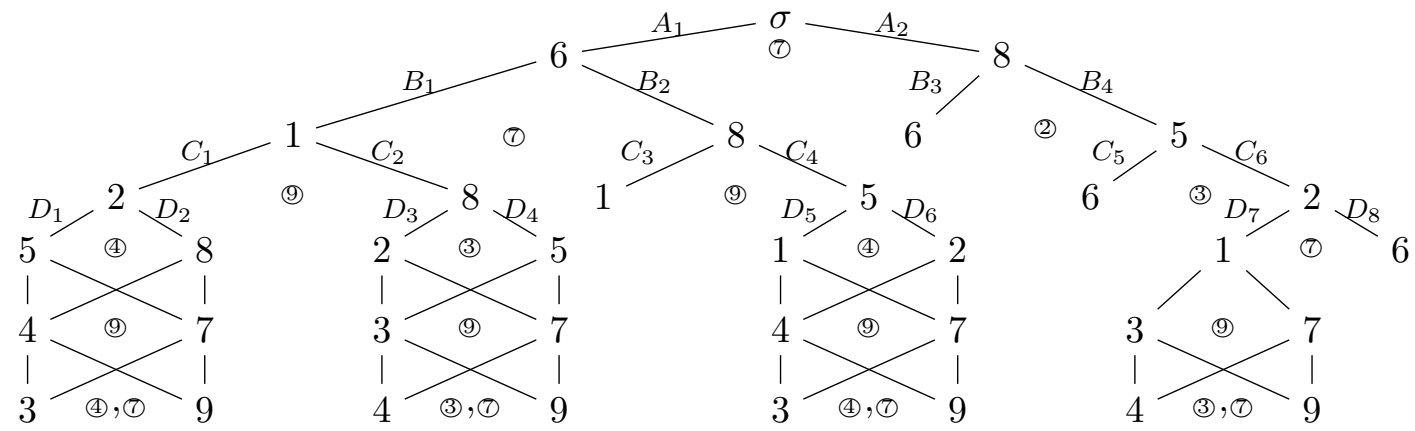

Note that the column to which two sub-nodes are merged to may not be unique. But it should not affect the final result. For example, at the very beginning we can merge the 6th and 8th column to either the 1 st or 7 th column. We will choose the 7 th column in the computation tree. On the other hand, if we go down the path $8-5-2-1-3$ then the 4 th and 9 th column can only be collapsed to the 7 th column since the 3 rd was removed already.

It turns out that even though $\mathfrak{s o}(7)$ and $\mathfrak{s o}(6)$ are not isomorphic Lie algebras, the computation of $\zeta_{W}(n, \mathfrak{s p}(6))$ is almost exactly the same as that of $\zeta_{W}(n, \mathfrak{s o}(7))$. By following the above computation tree we have

Theorem 5.1. Let $n$ be an even positive integer. Set $\left(\begin{array}{c}t \\ -1\end{array}\right)=0$ for all $t$. Then

$$
\begin{aligned}
\frac{48 \zeta_{W}(n, \mathfrak{s o}(7))}{(-720)^{n}(2 \pi)^{9 n}}=\frac{48 \zeta_{W}(n, \mathfrak{s p}(6))}{(-720)^{n}(2 \pi)^{9 n}} & =2^{n} \sum_{i=0}^{n}\left(\begin{array}{c}
2 n-i-1 \\
n-1
\end{array}\right)\left\{\sum_{j=0}^{i}\left(\begin{array}{c}
n+i-j-1 \\
n-1
\end{array}\right)\left(\frac{B(j)}{2^{i}}+\frac{B^{\prime \prime}(i, j)}{(-1)^{i}}\right)\right. \\
& \left.+\sum_{j=0}^{n}\left(\begin{array}{c}
n+i-j-1 \\
i-1
\end{array}\right)\left(\frac{B^{\prime}(j, n, j)}{2^{i}}+\frac{B^{\prime}(i+n, j, j)}{(-1)^{i+j}}\right)\right\},
\end{aligned}
$$

where by writing $C^{\prime}\{i, k\}=C^{\prime}(n+i, 3 n+i-k, 3 n+i-k, 2 n+i-k)$

$$
\begin{aligned}
B(j) & =\sum_{k=0}^{j}\left(\begin{array}{c}
n+j-k-1 \\
n-1
\end{array}\right) C(j, j-k, k, j)+\sum_{k=0}^{n}\left(\begin{array}{c}
n+j-k-1 \\
j-1
\end{array}\right) \frac{C(j, j-k, n, j)}{(-1)^{k}}, \\
B^{\prime}(a, b, j) & =\sum_{k=0}^{b}\left(\begin{array}{c}
n+j-k-1 \\
n+j-b-1
\end{array}\right) C(a, j-k, k, j)+\sum_{k=0}^{n+j-b}\left(\begin{array}{c}
n+j-k-1 \\
b-1
\end{array}\right) C^{\prime}(a, k, n, j), \\
B^{\prime \prime}(i, j) & =\sum_{k=0}^{2 n+i-j}\left(\begin{array}{c}
2 n+i-k-1 \\
j-1
\end{array}\right) \frac{2^{2 n+i-j-k} C^{\prime}\{i, k\}}{(-1)^{i-j-k}}+\sum_{k=0}^{j}\left(\begin{array}{c}
2 n+i-k-1 \\
2 n+i-j-1
\end{array}\right) \frac{2^{2 n+i-j} C^{\prime \prime}(i, k)}{(-1)^{i-j}},
\end{aligned}
$$


and by setting $\mu=n+a-j$ and $\nu=n+b-c$

$$
\begin{aligned}
C(a, b, c, j) & =\sum_{l=0}^{c}\left(\begin{array}{c}
n+a-b-l-1 \\
n+a-b-c-1
\end{array}\right) \frac{D(a, b, n, j, l)}{(-1)^{n+a-b-c}}+\sum_{l=0}^{n+a-b-c}\left(\begin{array}{c}
n+a-b-l-1 \\
c-1
\end{array}\right) \frac{D(a, b, n, j, l)}{(-1)^{n+a-b-c-l}}, \\
C^{\prime}(a, b, c, j) & =\sum_{l=0}^{\mu}\left(\begin{array}{c}
\mu+\nu-l-1 \\
\nu-1
\end{array}\right) D(a, b, c, j, l)+\sum_{l=0}^{\nu}\left(\begin{array}{c}
\mu+\nu-l-1 \\
\mu-1
\end{array}\right) D(a, b, c, j, l), \\
C^{\prime \prime}(i, k) & =\sum_{l=0}^{k}\left(\begin{array}{c}
n+k-l-1 \\
n-1
\end{array}\right) 2^{n} D^{\prime}(i, k, l)+\sum_{l=0}^{n}\left(\begin{array}{c}
n+k-l-1 \\
k-1
\end{array}\right) 2^{n-l} D(i+l-k, n, 3 n+i-k, 0, l),
\end{aligned}
$$

where by setting $u=4 n-a$ and $v=3 n+a+b-c-j-l$

$$
\begin{aligned}
D(a, b, c, j, l) & =\sum_{s=0}^{u}\left(\begin{array}{c}
u+v-s-1 \\
v-1
\end{array}\right) \frac{E(c, l, s)}{(-1)^{v}}+\sum_{s=0}^{v}\left(\begin{array}{c}
u+v-s-1 \\
u-1
\end{array}\right) \frac{E^{\prime}(c, l, s)}{(-1)^{v-s}}, \\
D^{\prime}(i, k, l) & =\sum_{s=0}^{4 n+k-i-l}\left(\begin{array}{c}
7 n-l-s-1 \\
3 n+i-k-1
\end{array}\right) \frac{E^{\prime \prime}(l, s)}{2^{7 n-l-s}}+\sum_{s=0}^{3 n+i-k}\left(\begin{array}{c}
7 n-l-s-1 \\
4 n+k-i-l-1
\end{array}\right) \frac{E^{\prime \prime}(l, s)}{2^{7 n-l-s}} .
\end{aligned}
$$

Here by setting $\beta_{s, t}=-B_{s} B_{t} B_{9 n-s-t} /(s ! t !(9 n-s-t) !), \beta_{a, b}^{\prime \prime}=\beta_{a, b}\left(1+\left(2^{1-a}-1\right)\left(2^{1-b}-1\right)\right) / 2$, and $\beta_{a, b, c}^{\prime \prime \prime}=\beta_{a, b}\left(1+\left(2^{1-a}-1\right)\left(2^{1-b}-1\right)\left(2^{1-c}-1\right)\right) / 2$, we have

$$
\begin{aligned}
E(c, l, s) & =\sum_{t=0}^{c}\left(\begin{array}{c}
c+s-t-1 \\
s-1
\end{array}\right) \frac{\beta_{l, t}}{2^{c+s-t}}+\sum_{t=0}^{s}\left(\begin{array}{c}
c+s-t-1 \\
c-1
\end{array}\right) \frac{\beta_{l, t}}{2^{c+s-t}}, \\
E^{\prime}(c, l, s) & =\sum_{t=0}^{c}\left(\begin{array}{c}
c+s-t-1 \\
s-1
\end{array}\right) \beta_{l, t}+\sum_{t=0}^{s}\left(\begin{array}{c}
c+s-t-1 \\
c-1
\end{array}\right) \beta_{l, t}, \\
E^{\prime \prime}(l, s) & =\sum_{t=0}^{n}\left(\begin{array}{c}
n+s-t-1 \\
s-1
\end{array}\right) \beta_{l, t, 9 n-l-t}^{\prime \prime \prime}+\sum_{t=0}^{s}\left(\begin{array}{c}
n+s-t-1 \\
n-1
\end{array}\right) \beta_{l, t}^{\prime \prime} .
\end{aligned}
$$

For example, Maple computation shows that

$$
\begin{aligned}
\zeta_{W}(2, \mathfrak{s o}(7)) & =\frac{2^{3} \cdot 19}{3^{3} \cdot 7 \cdot 17 !} \pi^{18}, \\
\zeta_{W}(4, \mathfrak{s o}(7)) & =\frac{2^{12} \cdot 307 \cdot 267743941589}{3 \cdot 7 \cdot 13 \cdot 19 \cdot 37 !} \pi^{36} \\
\zeta_{W}(6, \mathfrak{s o}(7)) & =\frac{2^{21} \cdot 2053 \cdot 9079132487 \cdot 265178091767}{3 \cdot 7 \cdot 11 \cdot 19 \cdot 54 !} \pi^{54} \\
\zeta_{W}(8, \mathfrak{s o}(7)) & =\frac{2^{29} \cdot 241 \cdot 40670746903 \cdot 36209034431567319455922705846157}{3 \cdot 5 \cdot 7 \cdot 13 \cdot 19 \cdot 74 !} \pi^{72}, \\
\zeta_{W}(10, \mathfrak{s o}(7)) & =\frac{2^{37} \cdot 61 \cdot 45197 \cdot 3920899 \cdot 3246046224154033 \cdot 202097025268393295809502658929}{3^{3} \cdot 7 \cdot 11 \cdot 19 \cdot 31 \cdot 89 !} \pi^{90} .
\end{aligned}
$$

When taking the sum over the range of $\left|m_{1}\right|,\left|m_{2}\right|,\left|m_{3}\right| \leq 100$ in (6.1) we find that $\zeta_{W}(2, \mathfrak{s o}(7))$ is correct up to at least 19 digits, $\zeta_{W}(4, \mathfrak{s o}(7))$ up to 42 digits, $\zeta_{W}(6, \mathfrak{s o}(7))$ up to 64 digits, $\zeta_{W}(8, \mathfrak{s o}(7))$ and $\zeta_{W}(10, \mathfrak{s o}(7))$ up to at least 80 digits. 
By definition

$$
\zeta_{W}(n ; \mathfrak{s l}(5))=288^{n} \sum_{m_{1}, \ldots, m_{4}=1}^{\infty}\left(\frac{1 /\left(m_{1} m_{2} m_{3} m_{4}\left(m_{1}+m_{2}\right)\left(m_{2}+m_{3}\right)\left(m_{3}+m_{4}\right)\right)}{\left(m_{1}+m_{2}+m_{3}\right)\left(m_{2}+m_{3}+m_{4}\right)\left(m_{1}+m_{2}+m_{3}+m_{4}\right)}\right)^{n}
$$

The corresponding matrix is

$$
\begin{aligned}
& \sigma=\left(\begin{array}{llllllllll}
1 & 0 & 0 & 0 & 1 & 0 & 0 & 1 & 0 & 1 \\
0 & 1 & 0 & 0 & 1 & 1 & 0 & 1 & 1 & 1 \\
0 & 0 & 1 & 0 & 0 & 1 & 1 & 1 & 1 & 1 \\
0_{n} & 0_{n} & 0_{n} & 1_{n} & 0_{n} & 0_{n} & 1_{n} & 0_{n} & 1_{n} & 1_{n}
\end{array}\right) \\
& \text { (1) (2) (3) (4) (5) (6) (1) 8) (9) }(8
\end{aligned}
$$

Here ${ }^{\otimes}$ is the 10 th column. The set of 3 -column dependencies is

$$
\{(1,2,5),(1,6,8),(1,9,10),(2,3,6),(2,7,9),(3,4,7),(3,5,8),(4,6,9),(4,8,10),(5,7,10)\} .
$$

So we have the following computation tree

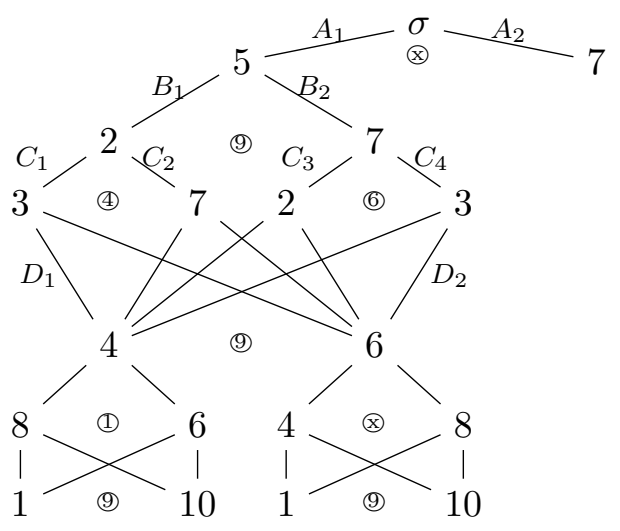

By symmetry $A_{1}=A_{2}$ so we get

Theorem 6.1. Let $n$ be a positive even integer. Set $\left(\begin{array}{c}t \\ -1\end{array}\right)=0$ for all $t$. Define $\beta_{s, t, k}=0$ if $s=1$ or $t=1$ or $k=1$ and define $\beta_{s, t, k}=(2 \pi)^{10 n} B_{s} B_{t} B_{k} B_{w-s-t-k} /(s ! t ! k !(w-s-t-k) !)$ for all other nonnegative integers $s, t, k$. Then

$$
\frac{60 \zeta_{W}(n, \mathfrak{s l}(5))}{288^{n}}=\sum_{i=0}^{n}\left(\begin{array}{c}
2 n-i-1 \\
n-1
\end{array}\right)\left(\sum_{j=0}^{i}\left(\begin{array}{c}
n+i-j-1 \\
n-1
\end{array}\right) B_{1}(i, j)+\sum_{j=0}^{n}\left(\begin{array}{c}
n+i-j-1 \\
i-1
\end{array}\right) B_{2}(i, j)\right)
$$


where for $\alpha=1,2$

$$
\begin{aligned}
B_{\alpha}(i, j) & =\sum_{k=0}^{j}\left(\begin{array}{c}
n+j-k-1 \\
n-1
\end{array}\right) C_{\alpha}(i, j, k)+\sum_{k=0}^{n}\left(\begin{array}{c}
n+j-k-1 \\
j-1
\end{array}\right) C_{\alpha}(i, j, k), \\
C_{\alpha}(i, j, k) & =\sum_{l=0}^{n}\left(\begin{array}{c}
3 n+j-k-l-1 \\
2 n+j-k-1
\end{array}\right) D_{\alpha}(i, k, l)+\sum_{l=0}^{2 n+j-k}\left(\begin{array}{c}
3 n+j-k-l-1 \\
n-1
\end{array}\right) D_{3-\alpha}(i, k, l), \\
D_{\alpha}(i, k, l) & =\sum_{s=0}^{l}\left(\begin{array}{c}
n+l-s-1 \\
n-1
\end{array}\right) E_{\alpha}(i, k, l, s)+\sum_{s=0}^{n}\left(\begin{array}{c}
n+l-s-1 \\
l-1
\end{array}\right) E_{\alpha}(i, k, l, s),
\end{aligned}
$$

and

$$
\begin{aligned}
& E_{1}(i, k, l, s)=\sum_{t=0}^{2 n+l-s}\left(\begin{array}{c}
5 n+l-i-s-t-1 \\
3 n-i-1
\end{array}\right) \beta_{s, t, k}+\sum_{t=0}^{3 n-i}\left(\begin{array}{c}
5 n+l-i-s-t-1 \\
2 n+l-s-1
\end{array}\right) \beta_{s, t, k}, \\
& E_{2}(i, k, l, s)=\sum_{t=0}^{n}\left(\begin{array}{c}
5 n+l-i-s-t-1 \\
4 n+l-i-s-1
\end{array}\right) \beta_{s, t, k}+\sum_{t=0}^{4 n+l-i-s}\left(\begin{array}{c}
5 n+l-i-s-t-1 \\
n-1
\end{array}\right) \beta_{s, t, k} .
\end{aligned}
$$

For example, we have

$$
\begin{aligned}
\zeta_{W}(2, \mathfrak{s l}(5)) & =\frac{1}{650970015609375} \pi^{20}=\frac{2^{16} \cdot 13}{3^{2} \cdot 5^{3} \cdot 7 \cdot 11 \cdot 18 !} \pi^{20}, \\
\zeta_{W}(4, \mathfrak{s l}(5)) & =\frac{2^{38} \cdot 1523 \cdot 2625375581}{3^{2} \cdot 5^{2} \cdot 7 \cdot 11 \cdot 41 !} \pi^{40}, \\
\zeta_{W}(6, \mathfrak{s l}(5)) & =\frac{2^{57} \cdot 30677 \cdot 2082905565627654787323001}{3^{2} \cdot 5^{2} \cdot 7 \cdot 11 \cdot 13 \cdot 31 \cdot 61 !} \pi^{60}, \\
\zeta_{W}(8, \mathfrak{s l}(5)) & =\frac{2^{79} \cdot 3^{2} \cdot 11 \cdot 85081 \cdot 1361779882876127669651 \cdot 728520415874861}{5^{2} \cdot 7 \cdot 17 \cdot 82 !} \pi^{80}, \\
\zeta_{W}(10, \mathfrak{s l}(5)) & =\frac{2^{98} \cdot 29 \cdot 13^{2} \cdot 2143 \cdot 6751027 \cdot 430667831149 a}{3^{2} \cdot 5^{2} \cdot 11 \cdot 17 \cdot 101 !} \pi^{100},
\end{aligned}
$$

where $a=201223346979560452521803194127591413$.

\section{REFERENCES}

[1] P.E. Gunnells and R. Sczech, Evaluation of Dedekind sums, Eisenstein cocycles, and special values of L-functions, Duke Math. J. 118 (2), (2003), 229-260.

[2] Y. Komori, K. Matsumoto and H. Tsumura, Zeta and L-functions and Bernoulli polynomials of root systems, Proc. Japan Acad. Ser. A 84 (2008), 57-62.

[3] Y. Komori, K. Matsumoto and H. Tsumura, On Witten multiple zeta-functions associated with semisimple Lie algebras II,II, preprint.

[4] Y. Komori, K. Matsumoto and H. Tsumura, On Witten multiple zeta-functions associated with semisimple Lie algebras $I V$, to appear in Glasgow Math J..

[5] Y. Komori, K. Matsumoto and H. Tsumura, Functional equations for zeta-functions of root systems. In preparation.

[6] K. Matsumoto and H. Tsumura, On Witten multiple zeta-functions associated with semisimple Lie algebras I, Annales de l'institut Fourier, 56 (5), (2006), 1457-1504.

[7] N. Nielsen, Die Gammafunktion. Band I. Handbuch der Theorie der Gammafunktion. Band II. Theorie des Integrallogarithmus und verwandter Transzendenten, Chelsea, New York, 1965.

[8] E. Witten, On quantum gauge theories in two-dimensions. Commun. Math. Phys. 141(1) (1991), $153-209$. 
[9] D. Zagier, Values of zeta function and their applications. Proc. of the First European Congress of Math. 2 (1994), $497-512$.

[10] J. Zhao and X. Zhou, Witten multiple zeta values attached to sl(4), arxiv: 0903.2383. Max-Planck Institute for Mathematics, MPIM2009-41

[11] J. Zhao, Alternating Euler sums and special values of Witten multiple zeta function attached to so(5). arXiv:math/0903.0473. Max-Planck Institute for Mathematics, MPIM2009-42

[12] J. Zhao, Multi-polylogs at twelfth roots of unity and special values of Witten multiple zeta function attached to the exceptional Lie algebra $G_{2}$. In press: J. of Alg. and Its Applications, 2010. arxiv: 0903.0888.

Department of Mathematics, Eckerd College, St. Petersburg, FL 33711, UsA

E-mail address: zhaoj@eckerd.edu

URL: http://home.eckerd.edu/ zhaoj 